\title{
A Nonlinear Integral Equation Related to Infectious Diseases
}

\author{
Mohamed Jleli $\mathbb{D}$ and Bessem Samet $\mathbb{D}$ \\ Department of Mathematics, College of Science, King Saud University, P.O. Box 2455, Riyadh 11451, Saudi Arabia \\ Correspondence should be addressed to Bessem Samet; bsamet@ksu.edu.sa
}

Received 24 October 2020; Revised 12 November 2020; Accepted 21 November 2020; Published 2 December 2020

Academic Editor: Tuncer Acar

Copyright (c) 2020 Mohamed Jleli and Bessem Samet. This is an open access article distributed under the Creative Commons Attribution License, which permits unrestricted use, distribution, and reproduction in any medium, provided the original work is properly cited.

In this paper, a nonlinear integral equation related to infectious diseases is investigated. Namely, we first study the existence and uniqueness of solutions and provide numerical algorithms that converge to the unique solution. Next, we study the lower and upper subsolutions, as well as the data dependence of the solution.

\section{Introduction}

We consider the nonlinear integral equation

$$
x(t)=\prod_{i=1}^{n}\left(f_{i}(t, x(t))+\int_{t-\tau_{i}}^{t} g_{i}(s, x(s)) d s\right), t \in \mathbb{R},
$$

where $n \geq 2$ is an integer and $\tau_{i}>0, i=1,2, \cdots, n$. In the case $n=1$ and $f_{1} \equiv 0,(1)$ reduces to

$$
x(t)=\int_{t-\tau_{1}}^{t} g_{1}(s, x(s)) d s t \in \mathbb{R} .
$$

The integral equation (2) models the spread of certain infectious diseases with periodic contact rate that varies seasonally (see [1]). Several results related to certain mathematical aspects of (2) have been obtained by many authors (see, e.g., [1-9] and the references therein). In particular, in [3], using the Picard operator technique, the integral equation (2) was investigated regarding the existence and uniqueness of solutions and periodic solutions, lower and upper subsolutions, the data dependence, and the differentiability of solutions with respect to a parameter.

In this paper, we are concerned with the integral equation (1). We first investigate the existence and uniqueness of solutions and provide numerical algorithms that converge to the unique solution. Next, we study the lower and upper subsolutions, as well as the data dependence of the solution.
The next section is devoted to the main results of this paper. Namely, in Subsection 2.1, we fix some notations that will be used throughout this paper. In Subsection 2.2, we provide some lemmas that will be used in the proofs of our main results. In Subsection 2.3, the existence and uniqueness of solutions and periodic solutions are derived using the Banach contraction principle. Moreover, an iterative algorithm based on Picard iteration for approximating the unique solution is provided. In Subsection 2.4, a Prešic ' -type iterative algorithm that converges to the unique solution is provided. Lower and upper subsolutions type results are obtained in Subsection 2.5. Finally, in Subsection 2.6, the data dependence of solutions is studied.

\section{Results}

We first fix some notations.

2.1. Notations. Let $I=[\alpha, \beta]$ and $J=[m, M]$, where $0<\alpha<\beta$ and $0<m<M$. Let

$$
\begin{gathered}
C(\mathbb{R} \times I, J)=\{f: \mathbb{R} \times I \longrightarrow J, f \text { is continuous }\}, \\
X=C(\mathbb{R}, I)=\{f: \mathbb{R} \longrightarrow I, f \text { is continuous }\} .
\end{gathered}
$$

The functional space $X$ is equipped with the norm $\|\cdot\|_{X}$, where 


$$
\|x\|_{X}=\sup _{t \in \mathbb{R}}|x(t)|, x \in X
$$

Notice that $\left(X,\|\cdot\|_{X}\right)$ is a Banach space.

2.2. Preliminaries. The following lemma will be useful later. It can be easily proved by induction.

Lemma 1. Let $\left\{a_{n}\right\}$ and $\left\{b_{n}\right\}$ be two real sequences. Then, for all $n \geq 2$,

$$
\begin{aligned}
& \left|a_{1} a_{2} \cdots a_{n}-b_{1} b_{2} \cdots b_{n}\right| \leq\left|a_{2} a_{3} \cdots a_{n}\right|\left|a_{1}-b_{1}\right| \\
& \quad+\left|b_{1}\right|\left|a_{3} a_{4} \cdots a_{n}\right|\left|a_{2}-b_{2}\right| \\
& \quad+\left|b_{1} b_{2}\right|\left|a_{4} a_{5} \cdots a_{n}\right|\left|a_{3}-b_{3}\right|+\cdots+\left|a_{n}\right|\left|b_{1} b_{2} \cdots b_{n-2}\right| \\
& \quad \cdot\left|a_{n-1}-b_{n-1}\right|+\left|b_{1} b_{2} \cdots b_{n-1}\right|\left|a_{n}-b_{n}\right| .
\end{aligned}
$$

We recall the following result due to Prešic ${ }^{\prime}[10]$.

Lemma 2. Let $(X, d)$ be a complete metric space, $k$ a positive integer and $\varphi: X^{k} \longrightarrow X$ a mapping satisfying the following condition:

$$
\begin{aligned}
& d\left(\varphi\left(x_{1}, x_{2}, \cdots, x_{k}\right), \varphi\left(x_{2}, x_{3}, \cdots, x_{k+1}\right)\right) \\
& \leq q_{1} d\left(x_{1}, x_{2}\right)+q_{2} d\left(x_{2}, x_{3}\right)+\cdots+q_{k} d\left(x_{k}, x_{k+1}\right),
\end{aligned}
$$

for all $x_{1}, \cdots, x_{k+1} \in X$, where $q_{1}, q_{2}, \cdots, q_{k}$ are nonnegative constants such that $q_{1}+q_{2}+\cdots+q_{k}<1$. Then,

(i) There exists a unique $x^{*} \in X$ such that

$$
x^{*}=\varphi\left(x^{*}, x^{*}, \cdots, x^{*}\right) .
$$

(ii) For all $x_{1}, x_{2}, \cdots, x_{k} \in X$, the sequence $\left\{x_{p}\right\} \subset X$ defined by

$$
x_{p+k}=\varphi\left(x_{p}, x_{p+1}, \cdots, x_{p+k-1}\right), p \geq 1
$$

is convergent to $x^{*}$.

For more details about the above result, we refer to [11-15].

2.3. Existence and Uniqueness Result. Problem (1) is investigated under the following conditions:

(C1) $f_{i}, g_{i} \in C(\mathbb{R} \times I, J), i=1,2, \cdots, n$.

(C2) For all $i=1,2, \cdots, n$, there exists a constant $L_{f_{i}}>0$ such that for all $t \in \mathbb{R}$,

$$
\left|f_{i}(t, u)-f_{i}(t, v)\right| \leq L_{f_{i}}|u-v|, u, v \in I \text {. }
$$

(C3) For all $i=1,2, \cdots, n$, there exists a constant $L_{g_{i}}>0$ such that for all $t \in \mathbb{R}$,

$$
\left|g_{i}(t, u)-g_{i}(t, v)\right| \leq L_{g_{i}}|u-v|, u, v \in I \text {. }
$$

(C4) $M^{n-1}\left(\prod_{i=1}^{n-1}\left(\tau_{i}+1\right)\right) \sum_{k=1}^{n}\left(L_{f_{k}}+L_{g_{k}} \tau_{k}\right)<1$.

(C5) $\alpha / m^{n} \leq \prod_{i=1}^{n}\left(\tau_{i}+1\right) \leq \beta / M^{n}$.

We have the following existence and uniqueness result.

Theorem 3. Under conditions $\left(C_{1}\right)-\left(C_{5}\right)$, problem (1) admits one and only one solution $x^{*} \in X$. Moreover, for all $x_{0} \in X$, the sequence $\left\{x_{p}\right\} \subset X$ defined by

$$
x_{p+1}(t)=\prod_{i=1}^{n}\left(f_{i}\left(t, x_{p}(t)\right)+\int_{t-\tau_{i}}^{t} g_{i}\left(s, x_{p}(s)\right) d s\right), t \in \mathbb{R}
$$

converges uniformly to $x^{*}$.

Proof. Let us define the operator $T: X \longrightarrow C(\mathbb{R}, \mathbb{R})$ by

$$
T(x)(t)=\prod_{i=1}^{n} T_{i}(x)(t), x \in X, t \in \mathbb{R},
$$

where

$$
T_{i}(x)(t)=f_{i}(t, x(t))+\int_{t-\tau_{i}}^{t} g_{i}(s, x(s)) d s, i=1,2, \cdots, n .
$$

By $\left(\mathrm{C}_{1}\right)$, for all $i=1,2, \cdots, n$ and $t \in \mathbb{R}$, one has

$$
T_{i}(x)(t) \leq M+\int_{t-\tau_{i}}^{t} M d s=\left(\tau_{i}+1\right) M,
$$

which yields

$$
T(x)(t) \leq M^{n} \prod_{i=1}^{n}\left(\tau_{i}+1\right) .
$$

Then, using $\left(\mathrm{C}_{5}\right)$, one deduces that

$$
T(x)(t) \leq \beta, t \in \mathbb{R} .
$$

Similarly, by $\left(\mathrm{C}_{1}\right)$, one has

$$
T_{i}(x)(t) \geq m+\int_{t-\tau_{i}}^{t} m d s=\left(\tau_{i}+1\right) m,
$$

which yields

$$
T(x)(t) \geq m^{n} \prod_{i=1}^{n}\left(\tau_{i}+1\right)
$$

Hence, using $\left(\mathrm{C}_{5}\right)$, one obtains

$$
T(x)(t) \geq \alpha, t \in \mathbb{R} .
$$


Therefore, it follows from (16) and (19) that

$$
T X \subset X
$$

Moreover, the set of solutions to the integral equation (1) coincides with the set of fixed points of the operator $T$. Next, by Lemma 1 , for all $x, y \in X$ and $t \in \mathbb{R}$, one has

$$
\begin{aligned}
& |T(x)(t)-T(y)(t)|=\left|\prod_{i=1}^{n} T_{i}(x)(t)-\prod_{i=1}^{n} T_{i}(y)(t)\right| \\
& \leq T_{2}(x)(t) T_{3}(x)(t) \cdots T_{n}(x)(t)\left|T_{1}(x)(t)-T_{1}(y)(t)\right| \\
& \quad+T_{3}(x)(t) T_{4}(x)(t) \cdots T_{n}(x)(t) T_{1}(y)(t)\left|T_{2}(x)(t)-T_{2}(y)(t)\right| \\
& \quad+T_{4}(x)(t) T_{5}(x)(t) \cdots T_{n}(x)(t) T_{1}(y)(t) T_{2}(y)(t) \\
& \quad \cdot\left|T_{3}(x)(t)-T_{3}(y)(t)\right|+\cdots+T_{1}(y)(t) T_{2}(y)(t) \cdots T_{n-1}(y)(t) \\
& \quad \cdot\left|T_{n}(x)(t)-T_{n}(y)(t)\right| .
\end{aligned}
$$

On the other hand, by $\left(\mathrm{C}_{2}\right)$ and $\left(\mathrm{C}_{3}\right)$, for all $i=1,2, \cdots, n$, one has

$$
\begin{aligned}
& \left|T_{i}(x)(t)-T_{i}(y)(t)\right| \leq\left|f_{i}(t, x(t))-f_{i}(t, y(t))\right| \\
& \quad+\int_{t-\tau_{i}}^{t}\left|g_{i}(s, x(s))-g_{i}(s, y(s))\right| d s \leq L_{f_{i}}|x(t)-y(t)| \\
& \quad+L_{g_{i}} \int_{t-\tau_{i}}^{t}|x(s)-y(s)| d s \leq\left(L_{f_{i}}+L_{g_{i}} \tau_{i}\right)\|x-y\|_{X} .
\end{aligned}
$$

Therefore, using (14), (21), and (22), one obtains

$|T(x)(t)-T(y)(t)| \leq M^{n-1}\left(\prod_{i=1}^{n-1}\left(\tau_{i}+1\right)\right) \sum_{k=1}^{n}\left(L_{f_{k}}+L_{g_{k}} \tau_{k}\right)\|x-y\|_{X}$,

which yields

$\|T x-T y\|_{X} \leq M^{n-1}\left(\prod_{i=1}^{n-1}\left(\tau_{i}+1\right)\right) \sum_{k=1}^{n}\left(L_{f_{k}}+L_{g_{k}} \tau_{k}\right)\|x-y\|_{X}, x, y \in X$.

Finally, using $\left(\mathrm{C}_{4}\right),(20)$ and $(24)$, the conclusion of the theorem follows from the Banach contraction principle.

Now, we consider problem (1) under the additional condition:

(C6) There exists $\omega>0$ such that for all $i=1,2, \cdots, n$,

$$
f_{i}(t+\omega, u)=f_{i}(t, u), g_{i}(t+\omega, u)=g_{i}(t, u), t \in \mathbb{R}, u \in I
$$

Theorem 4. Under conditions $\left(C_{1}\right)-\left(C_{6}\right)$, problem (1) admits one and only one $\omega$-periodic solution $x^{*} \in X$. Moreover, for any $\omega$-periodic function $x_{0} \in X$, the sequence $\left\{x_{p}\right\}$ defined by (11) converges uniformly to $x^{*}$.
Proof. Let $T: X \longrightarrow X$ be the operator defined by (12). Notice that from the proof of Theorem 3, we know that under conditions $\left(\mathrm{C}_{1}\right)-\left(\mathrm{C}_{5}\right)$, one has $T X \subset X$. Let $V$ be the closed subset of $X$ (with respect to the norm $\|\cdot\|_{X}$ ) defined by

$$
V=\{x \in X: x(t+\omega)=x(t), t \in \mathbb{R}\} .
$$

For all $x \in V$ and $t \in \mathbb{R}$, using $\left(\mathrm{C}_{6}\right)$, one obtains

$$
\begin{aligned}
T(x)(t+\omega) & =\prod_{i=1}^{n}\left(f_{i}(t+\omega, x(t+\omega))+\int_{t+\omega-\tau_{i}}^{t+\omega} g_{i}(s, x(s)) d s\right) \\
& =\prod_{i=1}^{n}\left(f_{i}(t, x(t))+\int_{t-\tau_{i}}^{t} g_{i}(\sigma+\omega, x(\sigma+\omega) d \sigma)\right) \\
& =\prod_{i=1}^{n}\left(f_{i}(t, x(t))+\int_{t-\tau_{i}}^{t} g_{i}(\sigma, x(\sigma)) d \sigma\right)=T(x)(t) .
\end{aligned}
$$

Hence, one has $T V \subset V$. On the other hand, since $V \subset X$, it follows from (24) that

$$
\|T x-T y\|_{X} \leq M^{n-1}\left(\prod_{i=1}^{n-1}\left(\tau_{i}+1\right)\right) \sum_{k=1}^{n}\left(L_{f_{k}}+L_{g_{k}} \tau_{k}\right)\|x-y\|_{X}, x, y \in V .
$$

Then, the conclusion of the theorem follows from the Banach contraction principle.

2.4. Prešic'-Type Approximation of the Unique Solution. Let us consider the integral equation (1) under conditions $\left(\mathrm{C}_{1}\right.$ )$-\left(C_{5}\right)$. Notice that by Theorem 3, (1) admits one and only one solution $x^{*} \in X$.

Theorem 5. Under conditions $\left(C_{1}\right)-\left(C_{5}\right)$, for any $x_{1}, x_{2}, \cdots$, $x_{n} \in X$, the sequence $\left\{x_{p}\right\}$ defined by

$$
\begin{aligned}
x_{p+n}(t)= & \left(f_{1}\left(t, x_{p}(t)\right)+\int_{t-\tau_{1}}^{t} g_{1}\left(s, x_{p}(s)\right) d s\right) \\
& \cdot\left(f_{2}\left(t, x_{p+1}(t)\right)+\int_{t-\tau_{2}}^{t} g_{2}\left(s, x_{p+1}(s)\right) d s\right) \cdots \\
& \cdot\left(f_{n}\left(t, x_{p+n-1}(t)\right)+\int_{t-\tau_{n}}^{t} g_{2}\left(s, x_{p+n-1}(s)\right) d s\right) \\
& p \geq 1, t \in \mathbb{R}
\end{aligned}
$$

converges uniformly to $x^{*}$.

Proof. Consider the function $\varphi: X^{n} \longrightarrow X$ defined by

$\varphi\left(x_{1}, x_{2}, \cdots, x_{n}\right)(t)=\prod_{i=1}^{n}\left(f_{i}\left(t, x_{i}(t)\right)+\int_{t-\tau_{i}}^{t} g_{1}\left(s, x_{i}(s)\right) d s\right), t \in \mathbb{R}$, 
that is,

$$
\varphi\left(x_{1}, x_{2}, \cdots, x_{n}\right)(t)=\prod_{i=1}^{n} T_{i}\left(x_{i}\right)(t)
$$

where for all $i=1,2, \cdots, n$, the operator $T_{i}$ is defined by (13). Notice that from the considered assumptions, one has $\varphi\left(X^{n}\right)$ $\subset X$, so $\varphi$ is well-defined. On the other hand, using Lemma 1 , for all $x_{1}, x_{2}, \cdots, x_{n}, x_{n+1} \in X$ and $t \in \mathbb{R}$, on has

$$
\begin{aligned}
\left|\varphi\left(x_{1}, x_{2}, \cdots, x_{n}\right)(t)-\varphi\left(x_{2}, x_{3}, \cdots, x_{n+1}\right)(t)\right| \\
=\left|\prod_{i=1}^{n} T_{i}\left(x_{i}\right)(t)-\prod_{i=1}^{n} T_{i}\left(x_{i+1}\right)(t)\right| \\
\leq T_{2}\left(x_{2}\right)(t) \cdots T_{n}\left(x_{n}\right)(t)\left|T_{1}\left(x_{1}\right)(t)-T_{1}\left(x_{2}\right)(t)\right| \\
\quad+T_{1}\left(x_{2}\right)(t) T_{3}\left(x_{3}\right)(t) \cdots T_{n}\left(x_{n}\right)(t)\left|T_{2}\left(x_{2}\right)(t)-T_{2}\left(x_{3}\right)(t)\right| \\
\quad+\cdots+T_{1}\left(x_{2}\right)(t) \cdots T_{n-1}\left(x_{n}\right)(t) \mid T_{n}\left(x_{n}\right)(t)-T_{n}\left(x_{n+1}(t) \mid .\right.
\end{aligned}
$$

Next, using (14), it holds that

$$
\begin{aligned}
& \left|\varphi\left(x_{1}, x_{2}, \cdots, x_{n}\right)(t)-\varphi\left(x_{2}, x_{3}, \cdots, x_{n+1}\right)(t)\right| \\
& \leq M^{n-1}\left(\prod_{i=1}^{n-1}\left(\tau_{i}+1\right)\right) \sum_{k=1}^{n} \mid T_{k}\left(x_{k}\right)(t)-T_{k}\left(x_{k+1}(t) \mid .\right.
\end{aligned}
$$

On the other hand, under the considered assumptions, for all $k=1,2, \cdots, n$, one has

$$
\mid T_{k}\left(x_{k}\right)(t)-T_{k}\left(x_{k+1}(t) \mid \leq\left(L_{f_{k}}+\tau_{k} L_{g_{k}}\right)\left\|x_{k}-x_{k+1}\right\|_{X} .\right.
$$

Hence, one deduces that

$$
\begin{aligned}
& \left\|\varphi\left(x_{1}, x_{2}, \cdots, x_{n}\right)-\varphi\left(x_{2}, x_{3}, \cdots, x_{n+1}\right)\right\|_{X} \\
& \leq M^{n-1}\left(\prod_{i=1}^{n-1}\left(\tau_{i}+1\right)\right) \sum_{k=1}^{n}\left(L_{f_{k}}+\tau_{k} L_{g_{k}}\right)\left\|x_{k}-x_{k+1}\right\|_{X} .
\end{aligned}
$$
follows.

Finally, using $\left(\mathrm{C}_{4}\right)$ and Lemma 2, the desired result 2.5. Lower and Upper Subsolutions. We consider problem (1) under conditions $\left(\mathrm{C}_{1}\right)-\left(\mathrm{C}_{5}\right)$. We recall that by Theorem 3 , problem (1) admits one and only one solution $x^{*} \in X$. We suppose also that

For all $i=1,2, \cdots, n$ and $t \in \mathbb{R}$, the functions

$$
f_{i}(t, \cdot): I \longrightarrow J \text { and } g_{i}(t, \cdot): I \longrightarrow J
$$

are nondecreasing.
Theorem 6. Suppose that conditions $\left(C_{1}\right)-\left(C_{5}\right)$ and $\left(C_{6}^{\prime}\right)$ are satisfied. If $x \in C(\mathbb{R}, I)$ satisfies

$$
x(t) \leq \prod_{i=1}^{n}\left(f_{i}(t, x(t))+\int_{t-\tau_{i}}^{t} g_{i}(s, x(s)) d s\right), t \in \mathbb{R},
$$

then

$$
x(t) \leq x^{*}(t), t \in \mathbb{R} \text {. }
$$

Proof. Let $T: X \longrightarrow X$ be the operator defined by (12). Then, (39) is equivalent to

$$
x(t) \leq T(x)(t), t \in \mathbb{R} .
$$

We shall prove that $T$ is a nondecreasing operator, that is, $u, v \in X, u(t) \leq v(t), t \in \mathbb{R} \Longrightarrow T(u)(t) \leq T(v)(t), t \in \mathbb{R}$

Let $u, v \in X$ be such that

$$
u(t) \leq v(t), t \in \mathbb{R} \text {. }
$$

By $\left(\mathrm{C}_{6}^{\prime}\right)$, for all $i=1,2, \cdots, n$ and $t \in \mathbb{R}$, one obtains

$0 \leq f_{i}(t, u(t))+\int_{t-\tau_{i}}^{t} g_{i}(s, u(s)) d s \leq f_{i}(t, v(t))+\int_{t-\tau_{i}}^{t} g_{i}(s, v(s)) d s$,

which yields

$$
\begin{aligned}
& \prod_{i=1}^{n}\left(f_{i}(t, u(t))+\int_{t-\tau_{i}}^{t} g_{i}(s, u(s)) d s\right) \\
& \leq \prod_{i=1}^{n}\left(f_{i}(t, v(t))+\int_{t-\tau_{i}}^{t} g_{i}(s, v(s)) d s\right),
\end{aligned}
$$

that is,

$$
T(u)(t) \leq T(v)(t)
$$

This proves (40). Next, by (39), it holds that

$$
x(t) \leq T(x)(t) \leq T^{2}(x)(t) \leq \cdots \leq T^{p}(x)(t),
$$

for all nonnegative integer $p$ and $t \in \mathbb{R}$, where

$$
T^{0}(x)(t)=x(t) \text { and } T^{p+1}(x)(t)=T\left(T^{p}(x)\right)(t) .
$$

Hence, it holds that

$$
x(t) \leq x_{p}(t), t \in \mathbb{R},
$$

where $\left\{x_{p}\right\}$ is the sequence defined by (11) with $x_{0}=x$. 
On the other hand, by Theorem 3, one has

$$
\lim _{p \rightarrow \infty} x_{p}(t)=x^{*}(t), t \in \mathbb{R} \text {. }
$$

Therefore, passing to the limit as $p \longrightarrow \infty$ in (47), (38) follows.

(A1) For $j=1,2,3$ and $i=1,2, \cdots, n$, let $f_{i}^{(j)}, g_{i}^{(j)} \in C(\mathbb{R}$ $\times I, J)$. We suppose that

(A2) For all $i=1,2, \cdots, n$ and $j=1,2,3$, there exists a constant $L_{f_{i}^{(j)}}>0$ such that for all $t \in \mathbb{R}$,

$$
\left|f_{i}^{(j)}(t, u)-f_{i}^{(j)}(t, v)\right| \leq L_{f_{i}^{(j)}}|u-v|, u, v \in I .
$$

For all $i=1,2, \cdots, n$ and $j=1,2,3$, there exists a constant $L_{g_{i}^{(j))}}>0$ such that for all $t \in \mathbb{R}$,

$$
\left|g_{i}^{(j)}(t, u)-g_{i}^{(j)}(t, v)\right| \leq L_{g_{i}^{(j)}}|u-v|, u, v \in I
$$

(A3) $M^{n-1}\left(\prod_{i=1}^{n-1}\left(\tau_{i}+1\right)\right) \sum_{k=1}^{n}\left(L_{f_{k}^{(j)}}+L_{g_{k}^{(j)}} \tau_{k}\right)<1, j=1,2$ , 3 .

(A4) $\alpha / m^{n} \leq \prod_{i=1}^{n}\left(\tau_{i}+1\right) \leq \beta / M^{n}$.

(A5) For all $i=1,2, \cdots, n$ and $t \in \mathbb{R}$, the functions

$$
f_{i}^{(2)}(t, \cdot): I \longrightarrow J \text { and } g_{i}^{(2)}(t, \cdot): I \longrightarrow J
$$

are nondecreasing.

(A6) For all $i=1,2, \cdots, n, t \in \mathbb{R}$ and $u \in I$,

$f_{i}^{(1)}(t, u) \leq f_{i}^{(2)}(t, u) \leq f_{i}^{(3)}(t, u)$ and $g_{i}^{(1)}(t, u) \leq g_{i}^{(2)}(t, u) \leq g_{i}^{(3)}(t, u)$.

Notice that by $\left(\mathrm{A}_{1}\right)-\left(\mathrm{A}_{4}\right)$, it follows from Theorem 3 that for all $j=1,2,3$, the integral equation

$$
x(t)=\prod_{i=1}^{n}\left(f_{i}^{(j)}(t, x(t))+\int_{t-\tau_{i}}^{t} g_{i}^{(j)}(s, x(s)) d s\right), t \in \mathbb{R},
$$

admits one and only one solution $x^{(j)} \in X$. Moreover, for all $j=1,2,3$ and $x_{0}^{(j)} \in X$, the sequence $\left\{x_{p}^{(j)}\right\} \subset X$ defined by

$x_{p+1}^{(j)}(t)=\prod_{i=1}^{n}\left(f_{i}^{(j)}\left(t, x_{p}^{(j)}(t)\right)+\int_{t-\tau_{i}}^{t} g_{i}^{(j)}\left(s, x_{p}^{(j)}(s)\right) d s\right), t \in \mathbb{R}$

converges uniformly to $x^{(j)}$.

Theorem 7. Under conditions $\left(A_{1}\right)-\left(A_{6}\right)$, one has

$$
x^{(1)}(t) \leq x^{(2)}(t) \leq x^{(3)}(t), t \in \mathbb{R} .
$$

Proof. For all $j=1,2,3$, let $T^{(j)}: X \longrightarrow X$ be the operator defined by

$$
T^{(j)}(x)(t)=\prod_{i=1}^{n}\left(f_{i}^{(j)}(t, x(t))+\int_{t-\tau_{i}}^{t} g_{i}^{(j)}(s, x(s)) d s\right), x \in X, t \in \mathbb{R} .
$$

From condition $\left(\mathrm{A}_{5}\right)$, the operator $T^{(2)}$ is nondecreasing, that is,

$u, v \in X, u(t) \leq v(t), t \in \mathbb{R} \Longrightarrow T^{(2)}(u)(t) \leq T^{(2)}(v)(t), t \in \mathbb{R}$

Moreover, by $\left(\mathrm{A}_{6}\right)$, one has

$$
T^{(1)}(u)(t) \leq T^{(2)}(u)(t) \leq T^{(3)}(u)(t), u \in X, t \in \mathbb{R} .
$$

Let $x_{0}^{(1)}, x_{0}^{(2)}, x_{0}^{(3)} \in X$ be such that

$$
x_{0}^{(1)}(t) \leq x_{0}^{(2)}(t) \leq x_{0}^{(3)}(t), t \in \mathbb{R}
$$

Hence, by (57), one obtains

$$
T^{(2)}\left(x_{0}^{(1)}\right)(t) \leq T^{(2)}\left(x_{0}^{(2)}\right)(t) \leq T^{(2)}\left(x_{0}^{(3)}\right)(t), t \in \mathbb{R} \text {. }
$$

On the other hand, by (58), one has

$$
\begin{gathered}
T^{(1)}\left(x_{0}^{(1)}\right)(t) \leq T^{(2)}\left(x_{0}^{(1)}\right)(t), t \in \mathbb{R}, \\
T^{(2)}\left(x_{0}^{(3)}\right)(t) \leq T^{(3)}\left(x_{0}^{(3)}\right)(t), t \in \mathbb{R} .
\end{gathered}
$$

Therefore, using (60), (61), and (62), one deduces that

$$
T^{(1)}\left(x_{0}^{(1)}\right)(t) \leq T^{(2)}\left(x_{0}^{(2)}\right)(t) \leq T^{(3)}\left(x_{0}^{(3)}\right)(t), t \in \mathbb{R},
$$

that is,

$$
x_{1}^{(1)}(t) \leq x_{1}^{(2)}(t) \leq x_{1}^{(3)}(t), t \in \mathbb{R}
$$

Repeating the same argument, by induction, one deduces that for all nonnegative integer $p$ and $t \in \mathbb{R}$,

$$
x_{p}^{(1)}(t) \leq x_{p}^{(2)}(t) \leq x_{p}^{(3)}(t)
$$

where $\left\{x_{p}^{(j)}\right\}, j=1,2,3$, is the sequence defined by (54). Finally, passing to the limit as $p \longrightarrow \infty$ in (65), the desired result follows.

2.6. Data Dependence of Solutions. Suppose that conditions $\left(C_{1}\right)-\left(C_{5}\right)$ are satisfied. Then, by Theorem 3 , the integral equation admits one and only one solution $x^{*} \in X$. Consider now the perturbed problem

$$
y(t)=\prod_{i=1}^{n}\left(F_{i}(t, y(t))+\int_{t-\tau_{i}}^{t} G_{i}(s, y(s)) d s\right), t \in \mathbb{R},
$$


where $F_{i}, G_{i} \in C(\mathbb{R} \times I, J), i=1,2, \cdots, n$. Suppose that $y^{*} \in X$ is a solution to the integral equation (66).

We have the following data dependence result.

Theorem 8. Suppose that for all $i=1,2, \cdots, n$, there exist $\sigma_{i}$, $\eta_{i}>0$ such that

$\left|f_{i}(t, u)-F_{i}(t, u)\right| \leq \sigma_{i},\left|g_{i}(t, u)-G_{i}(t, u)\right| \leq \eta_{i}, t \in \mathbb{R}, u \in I$.

Then,

$\left\|x^{*}-y^{*}\right\|_{X} \leq \frac{M^{n-1}\left(\prod_{i=1}^{n-1}\left(\tau_{i}+1\right)\right) \sum_{k=1}^{n}\left(\sigma_{k}+\tau_{k} \eta_{k}\right)}{\left[1-M^{n-1}\left(\prod_{i=1}^{n-1}\left(\tau_{i}+1\right)\right) \sum_{k=1}^{n}\left(L_{f_{k}}+L_{g_{k}} \tau_{k}\right)\right]}$.

Proof. Let

$$
S(y)(t)=\prod_{i=1}^{n} S_{i}(y)(t), t \in \mathbb{R},
$$

where

$$
S_{i}(y)(t)=F_{i}(t, y(t))+\int_{t-\tau_{i}}^{t} G_{i}(s, y(s)) d s, i=1,2, \cdots, n .
$$

Then, for all $t \in \mathbb{R}$, one has

$\left|x^{*}(t)-y^{*}(t)\right|=\left|T\left(x^{*}\right)(t)-S\left(y^{*}\right)(t)\right|=\left|\prod_{i=1}^{n} T_{i}\left(x^{*}\right)(t)-\prod_{i=1}^{n} S_{i}\left(y^{*}\right)(t)\right|$,

where the operator $T$ is defined by (12). Next, by Lemma 1 and (14), one obtains

$$
\begin{aligned}
& \left|x^{*}(t)-y^{*}(t)\right| \leq T_{2}\left(x^{*}\right)(t) \cdots T_{n}\left(x^{*}\right)(t)\left|T_{1}\left(x^{*}\right)(t)-S_{1}\left(y^{*}\right)(t)\right| \\
& \quad+S_{1}\left(y^{*}\right)(t) T_{3}\left(x^{*}\right)(t) \cdots T_{n}\left(x^{*}\right)(t)\left|T_{2}\left(x^{*}\right)(t)-S_{2}\left(y^{*}\right)(t)\right| \\
& \quad+\cdots+S_{1}\left(y^{*}\right)(t) \cdots S_{n-1}\left(y^{*}\right)(t)\left|T_{n}\left(x^{*}\right)(t)-S_{n}\left(y^{*}\right)(t)\right| \\
& \leq M^{n-1}\left(\prod_{i=1}^{n-1}\left(\tau_{i}+1\right)\right) \sum_{k=1}^{n}\left|T_{k}\left(x^{*}\right)(t)-S_{k}\left(y^{*}\right)(t)\right| .
\end{aligned}
$$

On the other hand, using $\left(\mathrm{C}_{2}\right)$ and $\left(\mathrm{C}_{3}\right)$ and (67), for all $k=1,2, \cdots, n$, one has

$$
\begin{aligned}
& \left|T_{k}\left(x^{*}\right)(t)-S_{k}\left(y^{*}\right)(t)\right| \leq\left|f_{k}\left(t, x^{*}(t)\right)-F_{k}\left(t, y^{*}(t)\right)\right| \\
& \quad+\int_{t-\tau_{k}}^{t}\left|g_{k}\left(s, x^{*}(s)\right)-G_{k}\left(s, y^{*}(s)\right)\right| d s \\
& \leq\left|f_{k}\left(t, x^{*}(t)\right)-f_{k}\left(t, y^{*}(t)\right)\right|+\left|f_{k}\left(t, y^{*}(t)\right)-F_{k}\left(t, y^{*}(t)\right)\right| \\
& \quad+\int_{t-\tau_{k}}^{t}\left|g_{k}\left(s, x^{*}(s)\right)-g_{k}\left(s, y^{*}(s)\right)\right| d s \\
& \quad+\int_{t-\tau_{k}}^{t}\left|g_{k}\left(s, y^{*}(s)\right)-G_{k}\left(s, y^{*}(s)\right)\right| d s \leq L_{f_{k}}\left\|x^{*}-y^{*}\right\|_{X} \\
& \quad+\sigma_{k}+L_{g_{k}} \tau_{k}\left\|x^{*}-y^{*}\right\|_{X}+\tau_{k} \eta_{k}=\left(L_{f_{k}}+L_{g_{k}} \tau_{k}\right)\left\|x^{*}-y^{*}\right\|_{X} \\
& \quad+\sigma_{k}+\tau_{k} \eta_{k} .
\end{aligned}
$$

Hence, by (72), it holds that

$$
\begin{aligned}
\left\|x^{*}-y^{*}\right\|_{X} \leq M^{n-1}\left(\prod_{i=1}^{n-1}\left(\tau_{i}+1\right)\right) \\
\cdot \sum_{k=1}^{n}\left[\left(L_{f_{k}}+L_{g_{k}} \tau_{k}\right)\left\|x^{*}-y^{*}\right\|_{X}+\sigma_{k}+\tau_{k} \eta_{k}\right],
\end{aligned}
$$

which yields

$$
\begin{aligned}
& {\left[1-M^{n-1}\left(\prod_{i=1}^{n-1}\left(\tau_{i}+1\right)\right) \sum_{k=1}^{n}\left(L_{f_{k}}+L_{g_{k}} \tau_{k}\right)\right]\left\|x^{*}-y^{*}\right\|_{X}} \\
& \leq M^{n-1}\left(\prod_{i=1}^{n-1}\left(\tau_{i}+1\right)\right) \sum_{k=1}^{n}\left(\sigma_{k}+\tau_{k} \eta_{k}\right) .
\end{aligned}
$$

Finally, by $\left(\mathrm{C}_{4}\right)$, the desired result follows.

\section{Data Availability}

No data were used to support this study.

\section{Conflicts of Interest}

The authors declare that they have no conflicts of interest.

\section{Authors' Contributions}

All authors contributed equally and significantly in writing this article. All authors read and approved the final manuscript.

\section{Acknowledgments}

The authors extend their appreciation to the Deputyship for Research and Innovation, Ministry of Education in Saudi Arabia for funding this research work through the project number IFKSURG-237. 


\section{References}

[1] K. L. Cooke and J. L. Kaplan, "A periodicity threshold theorem for epidemics and population growth," Mathematical Biosciences, vol. 31, no. 1-2, pp. 87-104, 1976.

[2] M. Dobritoiu and A. M. Dobritoiu, "An approximating algorithm for the solution of an integral equation from epidemics," Annali dell'Universita di Ferrara, vol. 56, no. 2, pp. 237-248, 2010.

[3] M. Dobritoiu, I. A. Rus, and M. A. Serban, "An integral equation arising from infectious diseases via Picard operators," Studia Universitatis Babeș-Bolyai Mathematica, vol. 52, pp. 81-94, 2007.

[4] D. Guo and V. Lakshmikantham, "Positive solutions of nonlinear integral equations arising in infectious diseases," Journal of Mathematical Analysis and Applications, vol. 134, no. 1, pp. 1-8, 1988.

[5] E. Kirr, "Existence of periodic solutions for some integral equations arising in infectious diseases," Studia Universitatis Babeș-Bolyai Mathematica, vol. 39, no. 2, pp. 107-119, 1994.

[6] R. Precup, "Monotone technique to the initial values problem for a delay integral equation from biomathematics," Studia Universitatis Babeș-Bolyai Mathematica, vol. 40, no. 2, pp. 63-73, 1995.

[7] I. A. Rus, "Fiber Picard operators theorem and applications," Studia Universitatis Babeș-Bolyai Mathematica, vol. 44, no. 3, pp. 89-97, 1999.

[8] R. Torrejon, "A note on a nonlinear integral equation from the theory of epidemics," Nonlinear Analysis: Theory, Methods \& Applications, vol. 14, no. 6, pp. 483-488, 1990.

[9] T. Trif, "Positive solutions of a nonlinear integral equation from biomathematics," Demonstratio Mathematica, vol. 32, pp. 129-138, 1999.

[10] S. B. Prešic, "Sur la convergence des suites," Comptes-rendus de l'Académie des Sciences de Paris, vol. 260, pp. 3828-3830, 1965.

[11] E. Ameer, H. Aydi, M. Arshad, H. Alsamir, and M. S. Noorani, "Hybrid multivalued type contraction mappings in $\alpha_{\mathrm{K}}$-complete partial b-metric spaces and applications," Symmetry, vol. 11, no. 1, p. 86, 2019.

[12] A. Babu, T. Došenovi, M. M. Ali, S. Radenovi, and K. P. Rao, "Some Prešic type results in b-dislocated metric spaces," Constructive Mathematical Analysis, vol. 2, no. 1, pp. 40-48, 2019.

[13] E. Karapinar, "A short survey on the recent fixed point results on b-metric spaces," Constructive Mathematical Analysis, vol. 1, no. 1, pp. 15-44, 2018.

[14] P. Patle, D. Patel, H. Aydi, and S. Radenovic, "ON H+type multivalued contractions and applications in symmetric and probabilistic spaces," Mathematics, vol. 7, no. 2, p. 144, 2019.

[15] C. Vetro, "A fixed-point problem with mixed-type contractive condition," Constructive Mathematical Analysis, vol. 3, no. 1, pp. $45-52,2020$. 